\section{P2-481 ACHIEVEMENTS OF THE ACTIVITIES TO CREATE HEALTHY LIFESTYLES BY HEALTHCARE PROFESSIONALS AND ADOLESCENT PEER LEADERS (PART 1)}

doi:10.1136/jech.2011.142976m.8

${ }^{1} \mathrm{H}$ Takamura, ${ }^{1} \mathrm{~S}$ Haruyama, ${ }^{1} \mathrm{R}$ Uehara, ${ }^{2} \mathrm{~S}$ Esumi, ${ }^{1} \mathrm{Y}$ Aoyama, ${ }^{1} \mathrm{E}-0$ Tsogzolbaatar, ${ }^{1} \mathrm{~A}$ Sadakane, 'I Chihara, ${ }^{1} \mathrm{~S}$ Tsuboi, ${ }^{1} \mathrm{Y}$ Nakamura. ${ }^{1}$ JIchi Medical University, Shimotsuke, Japan; ${ }^{2}$ Graduate of Human Sciences, Osaka University, Suita, Japan

Objectives As part of the grass-root technical cooperation program of the Japan International Cooperation Agency, we are working on the activities to create healthy lifestyles by healthcare professionals and adolescent peer leaders jointly with the Health Service Bureau of Veracruz State in the United Mexican States. This research aims to grasp people's knowledge and awareness about health in that state and to examine how the peer leader activities influenced the people of that state. The first report focuses on the differences in awareness of health between people living in an area introduced this activity and those in areas without it.

Method We conducted the survey in May to June 2010 with people of Veracruz sampled at random from the resident registers (aged 12-69) through the interview survey for hearing with question sheets to be recorded. We surveyed people's participation in the peer leader activities, their knowledge and awareness about health acquired through the activities, the changes in their life they recognised, and other matters.

Results 881 sheets were distributed, and 799 were collected with a collection rate of $90.7 \%$. Valid responses were 788 with a response rate of $89.4 \%$. In an area introduced the activity, $97 \%$ were always aware of health $(\mathrm{p}<0.001), 95 \%$ were willing to participate in health promotion campaigns $(\mathrm{p}<0.001)$, and $55 \%$ were interested in obesity $(\mathrm{p}<0.001)$.

Consideration The possibility that peer leader activities may have favourable effects on people's health awareness in that state has been shown

\section{P2-482 EVALUATING A SCHOOL BASED RCT TO REDUCE GENDER- BASED VIOLENCE AMONG HIGH SCHOOL STUDENTS IN KWAZULU-NATAL, SOUTH AFRICA (SA)}

\section{doi:10.1136/jech.2011.142976m.9}

${ }^{1} \mathrm{M}$ Taylor, ${ }^{*}{ }^{1} \mathrm{C}$ Jinabhai, 'S Dlamini, 'R Sathiparsad, 'A Meyer-Weitz, ${ }^{1} \mathrm{M}$ Eggers, ${ }^{2} \mathrm{H}$ de Vries. ${ }^{1}$ University of KwaZulu-Natal, Durban, KwaZulu-Natal, South Africa; ${ }^{2}$ University of Maastricht, Maastricht, The Netherlands

Gender-based violence is a public health problem in South Africa associated with increased risk of HIV infection. KwaZulu-Natal is the epicentre of the HIV epidemic in South Africa with youth of 15-24 years at high risk of infection. A school based RCT aimed to develop, implement and evaluate an intervention to reduce genderbased violence, defined as verbal (belittling), physical (hitting) and sexual (forced sex) abuse, among grade 9 students. Schools (16) were randomly selected in two Districts and trained facilitators implemented a 16 module classroom-based intervention based on prior elicitation research, in 8 experimental schools. Students completed a self-reporting questionnaire at T1 and 8 months later at T2. Of 764 students, $46.7 \%$ male, both sexes reported perpetrating violence and being abused verbally, physically and sexually. Although there was a reduction in verbal abuse (belittling boy/girlfriend) and physical abuse (hitting boy/girlfriend) after the intervention programme, a reduction in hitting was reported only by female students ( $\beta$ coefficient $0.16, p=0.025,95 \% \mathrm{CI}-0.047$ to 0.688 ). The study found that students who had attended the school-based intervention programme were three times less likely than the control group to report being forced to have sex ( $p=0.046$, OR $2.95,95 \%$ CI
1.02 to 8.53). These findings of a reduction in gender-based violence among students exposed to the intervention indicates that such a programme is useful for universal prevention efforts, but also highlights the importance of more focused attention on male students. In addition to school-based programmes there is a need for support at community level to reinforce school-based initiatives.

\section{P2-483 DENGUE IN BRAZIL: CURRENT SITUATION AND CHANGE IN EPIDEMIOLOGICAL PATTERN}

doi:10.1136/jech.2011.142976m.10

M G Teixeira, ${ }^{*}$ M D C N Costa, F R Barreto, M L Barreto. Federal University of Bahia, Salvador, Bahia, Brazil

Introduction Ae. aegypti, the commonest cause of Dengue fever in the world, was re-introduced to Brazil in the latter half of the 1970s. Favourable environmental conditions facilitated unchecked territorial expansion of this vector. This study describes the changing epidemiology of Dengue in Brazil 25 years after it re-emerged, exploring the main determinants of disease and outlining the implications for control.

Methods This study analysed serial case reports registered in Brazil since1986, describing the changing incidence and spatial distribution of Dengue.

Results Epidemic waves followed the emergence of each serotype (DENV 1-3), characterised by an increasing incidence (from 64.6 per 100000 in 1987 to 475.3 per 100000 in 2010) and severity of disease resulting in high case-fatality (14896 cases and 1212 deaths). In 2007, an important and sudden change in the age of individuals affected by dengue haemorrhagic fever was observed, with an increasing number of children affected.

Conclusion A change in the age distribution of incident cases must be due to the sequence of circulating serotypes of dengue virus in the population conferring different levels of herd immunity in different age groups. Dengue is a serious public health problem in Brazil. Difficulties controlling the vector in all countries highlights the need for the international scientific community to renew efforts to generate knowledge, allowing improvement and progress in the development of new tools and strategies for dengue prevention.

\section{P2-484 DIAGNOSIS OF SMEAR NEGATIVE PULMONARY TUBERCULOSIS IN LOW AND MIDDLE INCOME COUNTRIES}

\section{doi:10.1136/jech.2011.142976m.11}

${ }^{1} \mathrm{~J}$ Thoulass, * ${ }^{2} \mathrm{E}$ Katabira, ${ }^{3} \mathrm{~S}$ Kunustor, ${ }^{3} \mathrm{~J}$ Walley, ${ }^{4} \mathrm{M}$ Evans. ${ }^{1}$ Section of Population Health, University of Aberdeen, Aberdeen, UK; ${ }^{2}$ Faculty of Medicine, Makerere University, Kampala, Uganda; ${ }^{3}$ Nuffield Centre for International Health, University of Leeds, Leeds, UK; ${ }^{4}$ Department of Infectious Diseases, Ninewells Hospital and Medical School, Dundee, UK

Introduction Diagnosis in of pulmonary tuberculosis (PTB) in resource-limited settings relies heavily on detection of bacilli on sputum smears however HIV positive patients often have smearnegative disease. A new WHO diagnostic algorithm was developed to improve PTB diagnosis in HIV-prevalent resource-limited settings (integrated, expedited pathway with 2 rather than 3 smears in each set and no diagnostic antibiotic trial). We evaluated performance under operational conditions in a Ugandan district hospital.

Methods A prospective observational cohort study design was used. The existing "UgWHO03" and new "WHO07" diagnostic algorithms were evaluated over the first and second phases of the study respectively. New HIV patients were screened for PTB by a history of two or more weeks of cough. Diagnosis by algorithm was compared to gold standard mycobacterial culture. 
Results 90/147 and 132/166 of patients enrolled onto "UgWHO03" and "WHO07" respectively and were able to provide adequate sputum for culture. For UgWHO03: five cases of smear negative PTB and four cases of smear positive PTB diagnosed by algorithm and three cases by culture. For WHO07: 18 cases of smear negative PTB and seven cases of smear positive PTB diagnosed by algorithm and 1 case by culture. Sensitivity for smear-negative PTB increased nonsignificantly from $62.5 \%$ to $94.7 \% \quad(p=0.065)$, specificity was maintained ( $99 \%$ vs $98 \%$, not significant).

Conclusion Sample size was limited by the ability to provide sputum but there was a non-significant trend towards increased sensitivity which approached significance. This suggests that in the context of a more rapid pathway this algorithm may be beneficial.

\section{P2-485 IMMUNOGENICITY OF PANDEMIC INFLUENZA A (H1N1) MONOVALENT VACCINE IN PATIENTS ON LONG-TERM HAEMODIALYSIS}

doi:10.1136/jech.2011.142976m.12
Methods This study incorporates a review and meta-analysis of the literature on the relationship between temperature and morbidity. Utilising a number of different electronic databases, a comprehensive search was performed, the results of which were filtered against pre-defined selection criteria. Subgroups were defined according to type of morbidity and whether a heat or cold effect was examined. The studies were combined using both fixed and random effects models to examine both heterogeneity and the effect of a unit change in temperature.

Results 37 studies were selected to be included in the review. Studies only listing correlations were analysed separately from those reporting actual responses in morbidity per unit change in temperature. In particular, for hot effects an increase in $1^{\circ} \mathrm{C}$ resulted in an increased risk of morbidity (RR: $1.002,95 \%$ CI 1.001 to 1.003), and similarly for a $1^{\circ} \mathrm{C}$ decrease in temperature for cold effects $(R R$ : $1.003,95 \%$ CI 1.002 to 1.005 ).

Conclusion This study provides a thorough survey of the literature relating to the effects of temperature on morbidity. The mean effects of temperature were found from relevant studies, along with an examination of important subgroups.

\section{P2-487 POPULATION CHANGES, SOCIAL DISPARITIES AND CHILD HEALTH IN LATIN AMERICA}

doi:10.1136/jech.2011.142976m.14

Introduction Influenza is a potentially serious infection among haemodialysis (HD) patients, and the antibody response to vaccines among HD patients is thought to be weaker than that in healthy people. We conducted this study for the purpose of evaluating the immunogenicity and reactogenicity of the pandemic vaccine among patients on long-term HD.

Materials and Methods During the 2009-2010 influenza season, 170 HD patients (108 men, 62 women; mean age: $62.2 \pm 13.2$ years, mean and max duration of HD: 12.3 and 35 years, respectively) received two doses of monovalent inactivated unadjuvanted vaccine. Immunogenicity of the vaccine was evaluated according to conventionally used international criteria (EMEA, 1997; FDA, 2007). Results The geometric mean of HI titre 4 weeks after vaccination increased from a pre-vac level of 7.0 to 32.5 for the first injection, and to 37.8 for the second injection. The sero-conversion rate was $48 \%$ and sero-protection rate was $54 \%$ for the first injection, and $53 \%$ and $57 \%$ respectively for the second injection. The occurrence of systemic rush was significantly higher compared with healthy adults.

Conclusions A two-shot influenza vaccination series showed a booster effect and achieved the EMEA criteria at the first vaccination. No serious adverse events were detected, but systemic rush occurred frequently. A Dysfunction in detoxification ability due to the patients' renal disease may have caused this.

Funding This study was supported by a research grant for Research on Emerging and Re-emerging Infectious Diseases, Ministry of Health, Labor and Welfare, Japan.

\section{P2-486 ENVIRONMENTAL TEMPERATURE VARIATION AND MORBIDITY: A META-ANALYSIS}

doi:10.1136/jech.2011.142976m.13

L Turner, ${ }^{*}$ S Tong. Queensland University of Technology, Brisbane, Queensland, Australia

Introduction The relationship between temperature and mortality has been well documented in the literature, with studies examining the effects of both temperature variations and extremes. The impact of temperature on morbidity however has received far less attention. In particular, to date there has been no study of the existing literature examining the effect temperature has on morbidity.

M G Vega-Lopez, ${ }^{*}$ G J Gonzalez-Perez, P P Quintero-Vega. University of Guadalajara, Guadalajara, Mexico

Introduction America Latina -a region known for its social disparities- is currently experiencing a profound demographic and epidemiological transition. In such sense, this paper seeks to analyse the relationship between social inequalities, demographic changes and child health in Latin America.

Methods Information about population and health was obtained from diverse official sources; different statistics like ratios among extreme socioeconomic groups or countries (ordered in quartiles according to Human Development Index 2007) were calculated to quantify the difference in health indicators among countries or in selected countries.

Results Despite the observed population ageing in recent years, this study reveals that the population under 15 years old in Latin America in 2030 will be only slightly lower to the existent in 1990; furthermore, child population's will be increased in poorest countries of the region. Data reflects large differences in health indicators: in 2008 , infant mortality rate in quartile of the poorest countries almost triplicate the rate of the richest quartile; inside each studied country, notables differences related with poverty are also observed: in all the cases, the infant malnutrition rate or the $<5$ mortality rate are much higher in poorest population's quintile that in the richest quintile.

Conclusions The above-mentioned aspects evidence a dramatic inequity in child health -related to social disparities-, something more relevant since in absolute terms, Latin American population $<15$ will maintain similar levels in next decades and that in the poorest countries -or in the poorest regions in each country-child population's will be higher.

\section{P2-488 DENTAL CARIES IN A PORTUGUESE SAMPLE OF SCHOOLCHILDREN}

doi:10.1136/jech.2011.142976m.15

N Veiga, 0 Amaral, J Pereira, C Pereira.* Cl \& DETS, Health School, Polytechnic Institute of Viseu, Viseu, Portugal

Background Dental caries is the most prevalent disease worldwide. The objective of this study was to determine the mean of decayed/ 